\title{
Threshold for Induction Motor Terminal Transient Peak Voltage with Fast Switching Inverters
}

\author{
Martin Lindahl ${ }^{1,2}$, Erik Velander $^{2}$, Anders Blomberg $^{2}$, and Hans-Peter Nee ${ }^{1}$ \\ ${ }^{1}$ KTH Royal Institute of Technology, Stockholm, Sweden, Email: martlin@kth.se \\ ${ }^{2}$ Bombardier Transportation, Västerås, Sweden, Email: martin.lindahl@ rail.bombardier.com
}

\section{Acknowledgments}

The authors would like to thank the KTH Railway Group for the financial support of the work. The authors would also like to thank Florence Meier and Ann Persson for valuable contributions to the work.

\section{Keywords}

$\ll$ Silicon Carbide $(\mathrm{SiC}) \gg, \quad \ll$ Insulation $\gg, \quad \ll$ Switching speed》, $\ll$ Induction motor $\gg$, $\ll$ Asynchronous motor $\gg, \ll$ Adjustable speed drive $\gg, ~ «$ Rail vehicle $\gg, ~ \ll$ Traction application $\gg$, $\ll$ Industrial application》

\begin{abstract}
Concerns have been raised that motor voltages might be very high if the fast switching characteristics of silicon carbide ( $\mathrm{SiC}$ ) are to be utilized. For this reason, the magnitude of the terminal voltage of an induction motor is investigated when fed from a fast switching $\mathrm{SiC}$ inverter through a long cable. It is found that the main insulation demand for the $\mathrm{SiC}$ case is only increased by $5 \%$ compared to the silicon insulated-gate bipolar transistor (IGBT) case. This indicates that additional filters may not be necessary in applications with long cables where silicon IGBTs are replaced by fast switching SiC power semiconductors.
\end{abstract}

\section{Introduction}

Worldwide, energy systems are transformed at a rapid pace. The transformation is needed to modernize energy systems to be more sustainable and to limit emissions of carbon dioxide leading to climate change [1]. The energy efficiency must also be increased, reducing operating cost and delivering more utility for a given amount of energy production. In the transportation system electrified solutions such as electric traction for railway is an important part of a resource efficient and sustainable future. This transformation is enabled in part by the continuous improvements in the field of power electronics, where silicon carbide (SiC) power semiconductors is one of the promising emerging technologies.

The characteristics of $\mathrm{SiC}$, with lower conduction losses and switching losses compared to silicon $(\mathrm{Si})$ power semiconductors, make it suitable for future electrical drive solutions. SiC power semiconductors are improving in quality and have now reached a maturity level such that they can be used in real world conditions [2]. To achieve low switching losses, the semiconductor needs to be switched with a high switching speed. This in turn may lead to transients with high voltages on the electric motor, especially in applications with long supply cables [3]. Various proposals of how to model the switching behavior with long-cable-fed motors have been made in literature $[3,4,5,6]$. Repetitive high voltage pulses will decrease the lifetime of the insulation [7]. To reduce the peak voltage experienced by the insulation of the motor windings, filter solutions have been proposed $[4,5,8]$. The purpose of the filter is both to reduce the peak voltage during the transient and to increase the rise time of the voltage waveform at the 


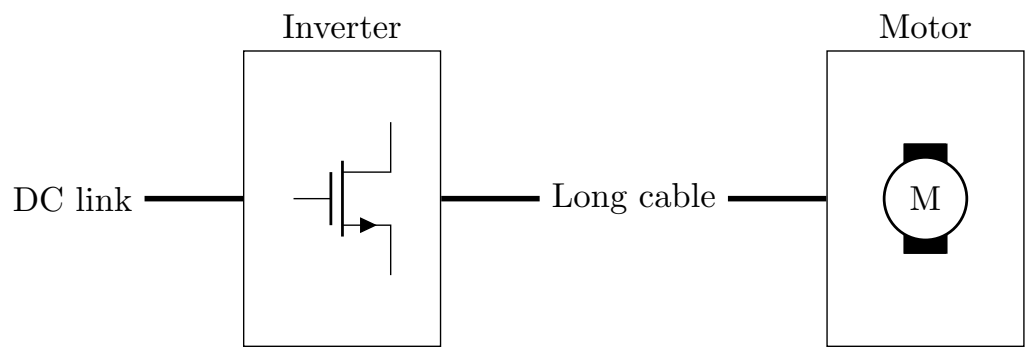

Fig. 1: Model overview. The inverter supplies a voltage pulse which is delivered to the induction motor through a long cable.

motor to decrease the turn-to-turn voltage in the motor windings. With an increased pulse amplitude on the motor terminal, also the turn-to-turn peak voltage will be increased. Concerns have been raised that the resulting motor voltages might be very high if the fast switching characteristics of $\mathrm{SiC}$ are to be utilized [9].

In electric drive applications for railway it is sometimes necessary to have long cables between the inverter and the motor. When Si gate turn-off thyristors (GTOs) were broadly used in railway drive systems, switching speeds below $1 \mathrm{kV} / \mu$ s were common. The introduction of $\mathrm{Si}$ insulated-gate bipolar transistors (IGBTs) made switching speeds in the range of $1-10 \mathrm{kV} / \mu$ s possible. Now, with $\mathrm{SiC}$ technology maturing, switching speeds above $10 \mathrm{kV} / \mu$ s are possible.

Based on transmission line theory, the theoretical maximum of the transient voltage is a doubling in amplitude when the incident wave is reflected. The investigations performed by Persson [3] indicated that the amplitude could increase by a factor 1.9 with long supply cables using Si IGBT technology. If these levels of voltage stress were reached already with Si IGBTs, only limited additional stress should result with fast switching SiC power semiconductors. Therefore, for some specific applications (for example electric traction for railway) the drawback might not be so severe. The hypothesis of the authors is, therefore, that the increase in voltage stress because of very fast switching with $\mathrm{SiC}$ power semiconductors is insignificant compared to Si IGBTs. In order to investigate this, this paper aims to use the model presented in [6] for calculations of the relation of transient voltage stress on the motor terminal with a broad interval of switching speeds, which are relevant for $\mathrm{SiC}$. To the best knowledge of the authors, such an investigation has not been performed previously.

In the following, first the modeling of the inverter, cable and motor will be described. Next, the conditions for the investigations and the results will be presented, and the paper will close with conclusions and discussion.

\section{High frequency modeling of cable and motor}

A simplified schematic diagram of the system which is modeled in this study is shown in Fig. 1. A voltage pulse is supplied from the inverter and starts to travel through the long cable, which is modeled with multiple cable segments. At the motor, a voltage reflection occurs with constructive interference as a consequence. From transmission line theory the reflection coefficient $\Gamma$ can be expressed as

$$
\Gamma=\frac{Z_{m}-Z_{c}}{Z_{m}+Z_{c}}
$$

where $Z_{c}$ is the characteristic impedances of the cable and $Z_{m}$ is the characteristic impedance of the motor. The resulting voltage on the motor terminal $V_{m}$, with the constructive interference, depends on the amplitude of the voltage pulse supplied by the inverter $\left(V_{i}\right)$ as 


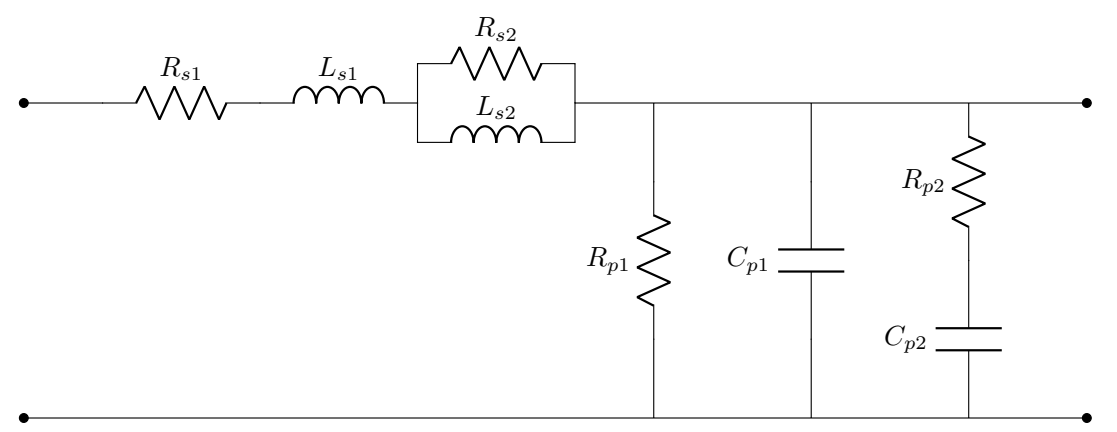

Fig. 2: Circuit representing a cable segment.

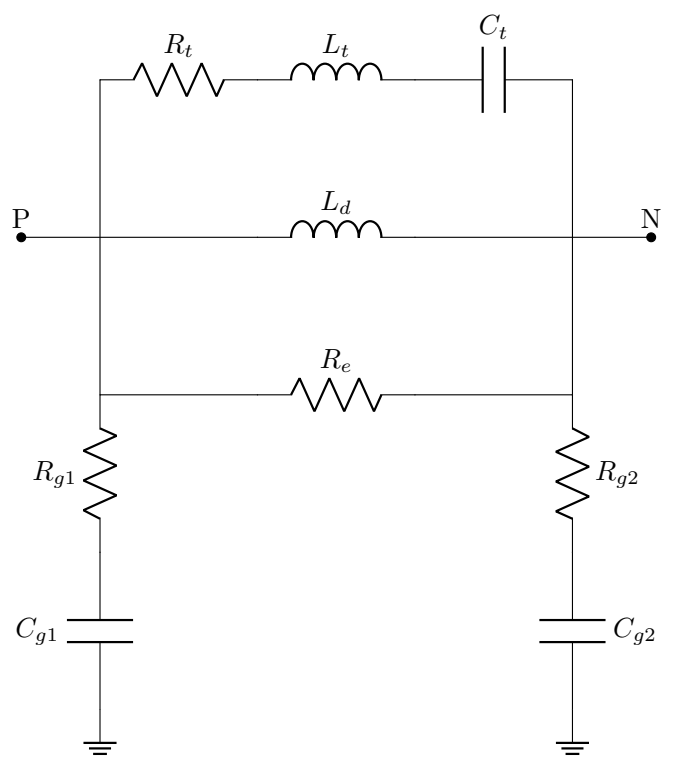

Fig. 3: Per phase motor equivalent circuit.

$$
V_{m}=(1+\Gamma) V_{i} .
$$

If a cable is terminated with its characteristic impedance no voltage reflection occurs. In this case (as is common for adjustable speed drive applications) the cable is not terminated with its characteristic impedance, so the voltage pulse is reflected at the induction motor and travels back towards the inverter. At the inverter the pulse is reflected again, but with opposite sign, and starts to travel back towards the motor. The result of this is an oscillation of the voltage at the motor terminal as was first described by Persson in 1992 [3].

In order to model the interactions of the inverter switching speed, the high-frequency phenomena in the long cable and in the induction motor must be modeled accurately. Wang et. al. proposed models for high-frequency modeling of cable segments as shown in Fig. 2 and induction motors as shown in Fig. 3 [6]. These models are adopted here to investigate the characteristics of the inverter-cable-motor system when the switching speed is increased orders of magnitude.

Parametrization of the models were performed in [6] for an ABB $2.2 \mathrm{~kW}$ ACS50 inverter with switching frequency of $16 \mathrm{kHz}$ and an ABB $2.2 \mathrm{~kW}$ induction motor with model number M2AA100LA. The rise and fall times of the inverter are stated to be $190 \mu$ s which should be a misprint since $190 \mathrm{~ns}$ is consistent with other information supplied in that paper. A rise time of $190 \mathrm{~ns}$ is used as the benchmark case here, for a cable length of $100 \mathrm{~m}$. 

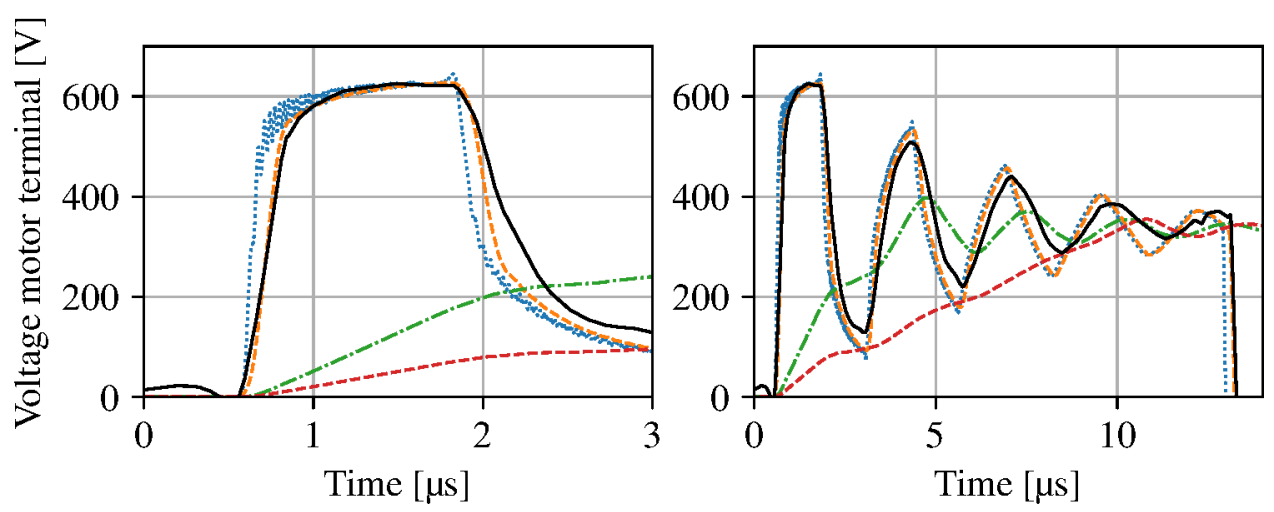

Fig. 4: Voltage transients on the motor terminal shown over two time ranges (same waveforms shown in both subplots). Simulated cases in dashed lines, measured data in solid line from [6] for reference. $\mathrm{rt}=$ rise time of the inverter pulse.

In [6] the modeling was performed with transmission-line modeling (TLM). In the present implementation, SPICE is instead used $[10,11]$. Experimental verification of the model was performed, and good agreement was obtained with the measurement results regarding the peak line-to-line voltage, delay time and oscillation frequency of the voltage measured at the motor terminal. Good agreement is achieved also with the SPICE implementation presented here.

The simulations are performed with a pulsed voltage source representing a pulse from the inverter in Fig. 1. Multiple cable segments according to Fig. 2 are put in sequence to model the cable. At the end of the cable the three-phase induction motor is modeled with the per-phase equivalent circuit according to Fig. 3 with one phase connected separately and returning with two phases connected in parallel. The rise time of the pulse from the inverter is varied and the results of this investigation are presented in the following section.

\section{Results}

With the inverter, cable and motor modeled with the high-frequency phenomena taken into account for improved accuracy, the rise time of the inverter pulse is varied over orders of magnitudes from $1 \mathrm{~ns}$ to $1 \mu \mathrm{s}$. The voltage pulse from the inverter has an amplitude of $335 \mathrm{~V}$ and the modeled length of the cable is $100 \mathrm{~m}$.

The resulting voltage on the motor terminal is shown for two time scales in Fig. 4. The same waveforms are shown in both subplots, where the different time scales are used to visualize how the shape of the voltage waveform varies for the different switching speeds. In the left subplot of Fig. 4 the first and largest voltage overshoot due to constructive interference can be seen (for the cases with high switching speed). In the right subplot the active part of the inverter pulse period is shown. It can be seen how the voltage pulse reflects back and forth between the motor and the inverter leading to oscillations in the motor terminal voltage, eventually settling around the inverter pulse amplitude. The frequency of the oscillation is determined by the cable length.

As seen in Fig. 4, the voltage transients can be classified in three groups depending on the rise time:

1. for long rise times (low switching speeds) the voltage increases linearly towards the inverter pulse amplitude virtually without overshoot,

2. for intermediate rise times the voltage increases towards its maximum value with a superimposed harmonic of the oscillation frequency, and

3. for short rise times the voltage quickly approaches a value slightly below the double of the inverter pulse amplitude (double due to the reflection and constructive interference).

The measured voltage for the case with $100 \mathrm{~m}$ cable from the experimental data reported in [6] is also shown in Fig. 4 for reference. 


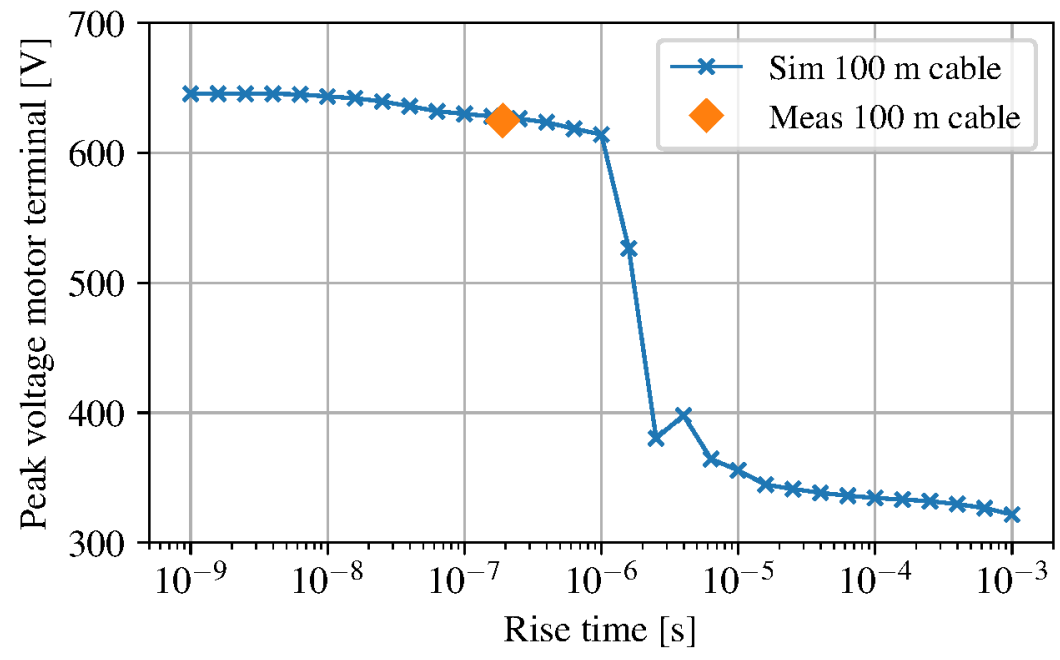

Fig. 5: Simulated peak values of the transient voltages at the motor terminal for a $100 \mathrm{~m}$ long cable and varying rise times. A benchmark from measurements in [6] is included.

In Fig. 5 the peak values of the transient voltages at the motor terminal have been extracted from the waveforms shown in Fig. 4 and from additional simulations. A step in peak voltage at the motor terminal can be observed when the rise time is decreased from approximately $10 \mu \mathrm{s}$ to $1 \mu \mathrm{s}$. Also shown is the benchmark case from measurement with $190 \mathrm{~ns}$ rise time for the $100 \mathrm{~m}$ long cable from [6]. One result, when looking at Fig. 5, is that the peak voltage on the motor terminal increases by approximately $5 \%$ when the rise time decreases from $1 \mu$ s to $1 \mathrm{~ns}$.

\section{Conclusion}

As the semiconductor technology has improved over the years, it has been possible to switch the semiconductor ever faster to reduce the switching losses. With the introduction of $\mathrm{SiC}$ as a potential semiconductor material in many power applications, and then with even higher potential switching speeds, there is again a need to investigate how and if the motor insulation can cope with the increasing switching speeds.

Interpreting the result shown in Fig. 5, it can be concluded that the stress on the main insulation due to high voltage to ground on the motor terminal only increases slightly by approximately $5 \%$ when the rise time decreases from $1 \mu \mathrm{s}$ to $1 \mathrm{~ns}$ for the investigated case. This is a very modest increase when the switching speed increases orders of magnitude. It should be mentioned that the result is from an interval of parameters for which the model has not yet been verified experimentally. In this sense the conclusion drawn here, based on the used model, can be seen as an hypothesis to be verified by future work.

Earlier, when the semiconductor technology improved so that relatively slow switching GTOs could be replaced by relatively fast switching IGBTs, then the "step" was taken to almost double the peak voltage on the motor terminal. For applications where this is valid, and since the step then already has been taken, only small increases in motor terminal peak voltage need to be accounted for when introducing $\mathrm{SiC}$ and utilizing its fast switching potential. This can potentially have large benefits in applications where otherwise filters would be needed, or where the semiconductor switching speed would have needed to be kept relatively low. To the best knowledge of the authors, this conclusion and its large potential benefits in applications has not been stated elsewhere.

Having said this, there is still a need to further investigate the effects of the high frequency behavior of the turn-to-turn voltages inside the motor. This will be the topic of future work.

\section{References}

[1] V. Masson-Delmotte, P. Zhai, H. O. Pörtner, D. Roberts, J. Skea, P. R. Shukla, A. Pirani, W. MoufoumaOkia, C. Péan, R. Pidcock, S. Connors, J. B. R. Matthews, Y. Chen, X. Zhou, M. I. Gomis, E. Lonnoy, 
T. Maycock, M. Tignor, and Waterfield, "Global warming of $1.5^{\circ} \mathrm{C}$. An IPCC special report on the impacts of global warming of $1.5^{\circ} \mathrm{C}$ above pre-industrial levels and related global greenhouse gas emission pathways, in the context of strengthening the global response to the threat of climate change," World Meteorological Organization, Geneva, Switzerland, Tech. Rep., 2018.

[2] M. Lindahl, E. Velander, M. H. Johansson, A. Blomberg, and H.-P. Nee, "Silicon carbide MOSFET traction inverter operated in the Stockholm metro system demonstrating customer values," in 2018 IEEE Vehicle Power and Propulsion Conference (VPPC), Aug. 2018, pp. 1-6.

[3] E. Persson, "Transient effects in application of PWM inverters to induction motors," IEEE Transactions on Industry Applications, vol. 28, no. 5, pp. 1095-1101, Sep. 1992.

[4] A. F. Moreira, P. M. Santos, T. A. Lipo, and G. Venkataramanan, "Filter networks for long cable drives and their influence on motor voltage distribution and common-mode currents," IEEE Transactions on Industrial Electronics, vol. 52, no. 2, pp. 515-522, Apr. 2005.

[5] A. von Jouanne and P. N. Enjeti, "Design considerations for an inverter output filter to mitigate the effects of long motor leads in ASD applications," IEEE Transactions on Industry Applications, vol. 33, no. 5, pp. 1138-1145, Sep. 1997.

[6] L. Wang, C. Ngai-Man Ho, F. Canales, and J. Jatskevich, "High-frequency modeling of the long-cable-fed induction motor drive system using TLM approach for predicting overvoltage transients," IEEE Transactions on Power Electronics, vol. 25, no. 10, pp. 2653-2664, Oct. 2010.

[7] F. J. T. E. Ferreira, G. Baoming, and A. T. de Almeida, "Reliability and operation of high-efficiency induction motors," IEEE Transactions on Industry Applications, vol. 52, no. 6, pp. 4628-4637, Nov. 2016.

[8] E. Velander, G. Bohlin, Å. Sandberg, T. Wiik, F. Botling, M. Lindahl, G. Zanuso, and H.-P. Nee, "An ultralow loss inductorless $d v / d t$ filter concept for medium-power voltage source motor drive converters with SiC devices," IEEE Transactions on Power Electronics, vol. 33, no. 7, pp. 6072-6081, Jul. 2018.

[9] P. Yi, P. K. S. Murthy, and L. Wei, "Performance evaluation of SiC MOSFETs with long power cable and induction motor," in 2016 IEEE Energy Conversion Congress and Exposition (ECCE), Sep. 2016, pp. 1-7.

[10] F. Salvaire, "PySpice v1.2.0," https://github.com/FabriceSalvaire/PySpice, 2018.

[11] H. Vogt, "ngspice v29," http://ngspice.sourceforge.net/, 2018. 